\title{
Screening strategies to identify new chemical diversity for drug development to treat kinetoplastid infections
}

\author{
ROB DON and JEAN-ROBERT IOSET* \\ Drugs for Neglected Diseases initiative, 15 Chemin Louis-Dunant, 1202 Geneva, Switzerland \\ (Received 4 Fune 2013; revised 18 Fuly 2013; accepted 22 Fuly 2013; first published online 28 August 2013) \\ SUMMARY
}

The Drugs for Neglected Diseases initiative (DND $i$ ) has defined and implemented an early discovery strategy over the last few years, in fitting with its virtual R\&D business model. This strategy relies on a medium- to high-throughput phenotypic assay platform to expedite the screening of compound libraries accessed through its collaborations with partners from the pharmaceutical industry. We review the pragmatic approaches used to select compound libraries for screening against kinetoplastids, taking into account screening capacity. The advantages, limitations and current achievements in identifying new quality series for further development into preclinical candidates are critically discussed, together with attractive new approaches currently under investigation.

Key words: High-throughput, high-content, screening, DND $i$, library selection, human African trypanosomiasis, Leishmaniasis, Chagas' disease.

\section{INTRODUCTION}

The Drugs for Neglected Diseases initiative $(\mathrm{DND} i)$ is a patients' needs-driven product development partnership created in 2003 which aims to address the lack of efficacious and safe treatments for neglected diseases. DND $i$ has adopted a pragmatic virtual $\mathrm{R} \& \mathrm{D}$ model operating in collaboration with partners from pharmaceutical as well as nonpharmaceutical sectors. The organization has a portfolio of research and development projects spanning the kinetoplastid infections (human African trypansomiasis, leishmaniasis and Chagas' disease), paediatric HIV infection, malaria and the filarial infections, onchocerciasis and lymphatic filariasis (www.dndi.org/diseases-projects/portfolio.html). At the research stage, DND $i$ concentrates its efforts on the establishment of a healthy and diverse pipeline for the filarial infections, human African trypanosomiasis, visceral leishmaniasis and Chagas' diseases, bearing in mind the inevitable attrition rate related to any R\&D programme (Ioset and Chang, 2011). This article will focus on strategies related to the screening of chemical libraries to identify novel lead series against the kinetoplastid parasites, Leishmania donovani (leishmaniasis) and Trypanosoma cruzi (Chagas' disease).

\section{SCREENING ASSAYS}

A major consideration for DND $i$ when developing its early discovery strategy has been the complexity

* Corresponding author: Drugs for Neglected Diseases initiative, 15 Chemin Louis-Dunant, 1202 Geneva, Switzerland. Tel: + 41 (0) 2290692 65. Fax: +41 (0) 22 90692 31. E-mail: jrioset@dndi.org of target validation (Sams-Dodd, 2005) and subsequent paucity of validated targets essential to the parasite which would be amenable for the development of assays allowing high-throughput screening in the field of neglected diseases (Wyatt et al. 2011). Indeed little has so far translated into R\&D breakthroughs despite huge expectations following the publication of the complete 'Tritryp' (Leishmania major, Trypanosoma brucei and T. cruzi) genome sequences in 2005 , revealing that each genome contains 8300-12000 protein-coding genes, of which around 6500 are common to all 3 genomes. It is fair to say that only a limited number of targets reviewed elsewhere (Renslo and McKerrow, 2006), mostly related to $T$. bruce $i$ sub-species, have so far reached a substantial level of biological characterization and validation in spite of the rapid development of the technologies and methodologies required for their assessment (Frearson et al. 2007). As far as kinetoplastid diseases are concerned, most of the drugs used in the field act via unclear or unknown mechanisms of action (Castillo et al. 2010). From the target-based assays implemented for screening purposes, few were able to deliver progressable candidates (Frearson et al. 2010) and none have led to new drugs or drug candidates in clinical research. This is partly due to a lack of translation from the target-based activity to whole-cell assays or in vivo activities. This observation is unfortunately not new to the field of antiinfective drug discovery, with similar hopes raised from the first publication of the bacterial genome in 1995, which triggered pharmaceutical companies to move back to antibacterial research and development using genomics-derived, target-based strategies to screen for new classes of drugs with novel modes of action. Despite the evaluation of more than 
300 genes, together with 70 high-throughput screening campaigns at Glaxo SmithKline (GSK) over a period of 7 years, there was a very modest yield. A mere 16 campaigns were able to deliver hits and only 5 of these could be turned into leads (Payne et al. 2007). Lessons learnt from this, and other specific analyses have dramatically influenced antibacterial drug discovery strategies within pharmaceutical companies over the last decade. GSK took the strategic decision to rely as early as possible on in vitro phenotypic assays and in vivo models to characterize the biological activity of the molecules under investigation, together with the use of HTS whole-cell assays for screening. Similarly, Product Development Partnerships involved in drug discovery activities for malaria (MMV), tuberculosis (TB Alliance) and kinetoplastids (DND $i$ ) have opted for medium- to high-throughput screening based on whole-cell assays to evaluate compound libraries accessed from their partners for hit identification. This cell-based screening approach has already been successfully used to identify several novel clinical candidates including thiazolidinones, spiroindolones and diarylquinolines for the treatment of hepatitis $\mathrm{C}$, malaria and tuberculosis respectively, as recently reviewed by Keller et al. (2011).

The intracellular amastigotes of $L$. donovani and T. cruzi in the host cells of infected tissues are the focus of drug discovery efforts. Because of the relative failure of target-based screening in anti-infective drug discovery, since 2008 DND $i$ has strategically invested in the development and validation of highcontent phenotypic screening assays against the intracellular stages of these parasites, in partnership with Institut Pasteur Korea and the University of Dundee. These assays are based on imaging analysis technology that specifically differentiates between infected and non-infected cells via the spatial localization and segmentation of specific descriptors related to the host cell and the parasites. The development of those high-content screening assays against L. donovani (Siqueira-Neto et al. 2012) and T. cruzi, in combination with an increased 384-well throughput capacity against the extracellular Trypanosoma brucei brucei at the Eskitis Institute for Cell and Molecular Therapies, Griffith University (Sykes and Avery, 2009), have enabled the screening of medium (10000-50000) to large size (50000$200000)$ commercial and proprietary libraries accessed by DND $i$. To date, this screening platform has allowed evaluation of more than one million compounds, leading to the identification of numerous novel active series against all three kinetoplastids, many of which have been, or are currently being, progressed in the lead optimization phase. In addition to a dramatic increase in screening throughput against the intracellular amastigote form of the pathogens, high-content assays are also more informative and more specific than colorimetric or fluorimetric assays. As such, it is possible to discriminate between activity resulting from specific pathogen killing versus activity due to host cell damage, or any sub-cellular event possibly indicative of a specific mechanism of action. Such additional image analysis information can be used immediately after screening or archived in anticipation of any subsequent complementary analysis.

SELECTION OF CHEMICAL LIBRARIES: DNDI'S APPROACHES

The introduction of automated high-content screening has led to very significant increases in screening throughput of intracellular Leishmania spp. and $T$. cruzi amastigotes over the last five years. Nevertheless, compared to the classic throughput achieved in pharmaceutical companies, with a capacity for tens of thousands of screening points per month, it can still only be considered as a medium-throughput screen. Therefore judicious selection and prioritization of chemical libraries is necessary to take advantage of the still limited capacity.

DND $i$ collaborates closely with its industrial partners, and has built new research partnerships with a number of pharmaceutical and biotechnology companies over the last 18 months in order to access chemical diversity (Ioset and Chang, 2011). Not only does this enable access to quality chemical libraries, but also, very importantly, to well annotated analogues after identification of hits from primary screens of small representative libraries. Most of our research collaborations have come with initial restrictions on publication of structural data from primary screens. Nonetheless, in line with DNDi's policy of open access, we are in positive negotiations with most partners from the pharmaceutical industry to make structures and annotation available as hits progress to lead optimization programmes, following the DND $i$ internally defined criteria and proposed cut-off values to qualify at the hit and lead selection stages (see Tables 1 and 2). It is worth noting that these are not applied strictly but are used to guide and prioritize hit and lead series, with the global activities, toxicities and chemical profiles being taken into account. The criteria and values listed are reviewed on a regular basis and are therefore subject to change.

Having gained access to considerable chemical diversity from the pharmaceutical industry, it has been necessary to carefully select or request specific libraries. Complete files belonging to large pharmaceutical companies are in the order of several million compounds and, as such, are still beyond the capacity for screening against intracellular amastigotes. The main strategies adopted by DND $i$ for library selection are outlined below.

Indication/repositioning libraries are small files of a few hundred compounds which have progressed to preclinical or clinical development for a variety 
Table 1. Criteria at hit stage

\begin{tabular}{|c|c|c|c|c|}
\hline Criteria & Parameter & Hit HAT & Hit VL & Hit Chagas \\
\hline \multirow[t]{2}{*}{ Efficacy in vitro } & Activity & $\begin{array}{l}\text { IC50 } \leqslant 10 \mu \mathrm{M} \\
T . b . \text { brucei } \\
\text { sub-species } \\
\text { bloodstream form }\end{array}$ & $\begin{array}{l}\text { IC } 50 \leqslant 10 \mu \mathrm{M} \\
\text { L. donovani or } \\
\text { L. infantum } \\
\text { intracellular } \\
\text { amastigote assav }\end{array}$ & $\begin{array}{l}\text { IC50 } \leqslant 10 \mu \mathrm{M} \\
T . \text { cruzi intracellular } \\
\text { amastigote assay TcVI } \\
\text { (Tulahuen) or TcII/Y strain }\end{array}$ \\
\hline & Selectivity & \multicolumn{3}{|c|}{10 fold more active $v s$ mammalian cell line } \\
\hline \multirow[t]{2}{*}{ DMPK in silico } & Lipinski's rules & \multicolumn{3}{|c|}{$\begin{array}{l}\text { Used to prioritize hits but should not be used as definitive selection criteria } \\
\text { (drug-likeness preferable but not essential) }\end{array}$} \\
\hline & $\begin{array}{l}\text { Structural alerts } \\
\text { (metabolism/stability/ } \\
\text { reactivity) }\end{array}$ & \multicolumn{3}{|c|}{ Proceed with caution and assay asap to determine extent of perceived problem } \\
\hline Toxicity in silico & Structural alerts & \multicolumn{3}{|c|}{ Proceed with caution and assay asap to determine extent of perceived problem } \\
\hline Chemical profile & Chemical tractability & \multicolumn{3}{|c|}{ Acceptable synthetic pathway for compound and/or analogues ( $<8$ steps) } \\
\hline & Stereo-chemistry & \multicolumn{3}{|c|}{ Assessed as part of chemical tractability } \\
\hline
\end{tabular}

of indications, with some compounds already registered as drugs. Screening such libraries could be considered to be very much a lottery, where the chance of success is very low but a positive hit would be associated with considerable annotation associated with a drug discovery programme. Unfortunately, no promising hits have yet been identified from screens of indication sets sponsored by DND $i$.

A similar high-risk but potentially high-return strategy has been the screening of libraries which address specific pharmacokinetic, absorption, distribution, metabolism and excretion (PK/ADME) criteria. These include compounds which are orally available or which cross the blood-brain barrier, essential for drugs intended to treat human African trypanosomiasis. It is expected that any active compounds would qualify for, or be close to qualifying for, in vivo efficacy testing, and may also be related to a well characterized family of compounds held by the partner from the pharmaceutical sector. A small number of hits have been identified in these screens. In all cases, the human targets against which these compounds were developed did not have an equivalent parasitic target, and it is probable that the mechanism of action in the parasite is different.

Chemical classes with known anti-infective properties are the third class of compounds we selected for screening. These have included macrolides, fluoroquinolones, oxazolidinones, hydrazones, ribosome inhibitors and anti-fungal compounds amongst many other proprietary anti-infective compounds. None have so far yielded hits which have progressed past early lead optimization efforts. This probably reflects the absence or genotypic divergence of the kinetoplastid target from that in the infectious agents against which the original pharmaceutical research was directed. This can be illustrated by the differences in phylogenetic lineage and functional homology of DNA topoisomerase II from eukaryotic kinetoplastids as compared to other eukaryotic cells or prokaryotic bacteria, the latter being the target of quinolone antibiotics (Das et al. 2004). It should be noted, however, that this collaborative screening approach has not been exhaustively completed with the relevant pharmaceutical and biotechnology companies.

DND $i$ has sponsored a small number of phenotypic screens with target-based libraries such as protease inhibitors and kinase inhibitors. In general they have been avoided because of a lack of a thorough validation of these targets in spite of the considerable efforts of drug discovery directed towards them during the last few decades (Renslo and McKerrow, 2006; McKerrow et al. 2008; Chawla and Madhubala, 2010). In addition, it is also likely that compounds with specificity towards the human orthologues of these targets will display reduced activity against the parasite target. This potential issue could eventually be addressed in the lead optimization phase in close collaboration with a pharmaceutical company via a selection of pre-existing moderately active or inactive analogues against the human target, as well as through the selective synthesis of analogues using in silico modelling tools supported by a counter screen on a human kinase panel.

Finally, diversity libraries have been selected as a source of new leads, such as the Pfizer Global Diversity Research Set which consists of 150000 compounds representative of the global Pfizer 3.5 million collection (Bell et al. 2013). Several additional core diversity sets have also been accessed and screened by DND $i$ as part of ongoing collaborations with pharmaceutical companies. DND $i$ expects to identify most of their new compound classes for development from these and similar libraries, although low hit rates, lack of R\&D background knowledge and IP encumbrance may present obstacles along the way. The global hit rate of so-called unbiased diversity sets is lower than $0 \cdot 1 \%$ (ranging from 0.02 to $0 \cdot 09 \%$ ) when screening against the intra-macrophagic 
Table 2. Criteria at lead stage

\begin{tabular}{|c|c|c|c|c|}
\hline Criteria & Parameter & Lead HAT & Lead VL & Lead Chagas \\
\hline \multirow[t]{2}{*}{ Efficacy in vitro } & Activity & $\begin{array}{l}\text { IC } 50 \text { 10-20-fold increase in } \\
\text { activity over Hit Active against } \\
T . \text { b. rhodesiense and/or } \\
\text { T. b. gambiense sub-species }\end{array}$ & $\begin{array}{l}\text { IC50 } \leqslant 1 \mu \mathrm{M} \text { IC } 50 \leqslant 1 \mu \mathrm{M} \\
\text { Active against a panel of } \\
\text { clinical isolates (Indian, } \\
\text { Soudanese) and } \\
\text { antimonial-resistant strain }\end{array}$ & $\begin{array}{l}\text { IC50 } 10-20 \text {-fold increase in } \\
\text { activity over Hit }\end{array}$ \\
\hline & Selectivity & \multicolumn{3}{|c|}{ Increased over Hit (ideally $\geqslant 50$-fold) more active vs. mammalian cell line } \\
\hline Efficacy in vivo & & $\begin{array}{l}\text { Significant reduction in parasitaemia and/or } \\
\text { increase in life-span }\left(4 \times 50 \mathrm{mg} \mathrm{kg}^{-1}\right) \text { i.p or } \\
\text { p.o in acute model }\end{array}$ & $\begin{array}{l}>70 \% \text { reduction in } \\
\text { liver parasitaemia after } \\
\text { i.p administration }\end{array}$ & $\begin{array}{l}80 \% \text { parasitaemia reduction } \\
\text { or no parasites detected at the } \\
\text { end of treatment and increase } \\
\text { in life-span }\left(10 \times 50 \mathrm{mg} \mathrm{kg}^{-1} \text { p.o. }\right)\end{array}$ \\
\hline DMPK in silico & Lipinski's rules & \multicolumn{3}{|c|}{ Used to prioritize hits but should not be used as definitive selection criteria (drug-likeness preferable but not essential) } \\
\hline DMPK in vitro & $\begin{array}{l}\text { Metabolic stability } \\
\text { (mouse and human (S9) liver } \\
\text { microsomes) }\end{array}$ & \multicolumn{3}{|c|}{ Stability - progress compounds ranked 'medium' or 'high' in assays (e.g. Clint $<50 \mathrm{~mL} \mathrm{~min}^{-1} \mathrm{~kg}^{-1}, \mathrm{t} 1 / 2>30 \mathrm{~min}$ ) } \\
\hline & $\begin{array}{l}\text { Caco } 2 \text { or MDCK Pgp } \\
\text { cell permeability }\end{array}$ & $\begin{array}{l}\text { Progress compounds ranked 'medium' } \\
\text { or 'high' in assays }\end{array}$ & \multicolumn{2}{|l|}{ Not required } \\
\hline & Solubility & \multicolumn{3}{|c|}{ Lowest value at $\mathrm{pH} 7.4>0.01 \mathrm{mg} \mathrm{mL}^{-1}$} \\
\hline & Protein binding & \multicolumn{3}{|c|}{ Only measured if perceived problem with bioavailability } \\
\hline & CYP3A4 inhibition & \multicolumn{3}{|c|}{$\mathrm{IC} 50>10 \mu \mathrm{M}$} \\
\hline & Plasma and whole blood stability & \multicolumn{3}{|l|}{ Measured } \\
\hline DMPK in vivo & Mouse PK & \multicolumn{2}{|c|}{$\begin{array}{l}\text { Plasma concentration in the range of active concentrations observed after } \\
\text { i.p administration }\end{array}$} & $\begin{array}{l}\text { Mouse exposure at } 10 \mathrm{mg} \mathrm{kg}^{-1} \text { bid } \\
\text { or } 20 \mathrm{mg} \mathrm{k}^{-1} \mathrm{qd} \text {. compounds which } \\
\text { achieve plasma levels above IC } 50 \text { for at } \\
\text { least } 24 \mathrm{~h} \text { are progressed in vivo }\end{array}$ \\
\hline Toxicity in vitro & Presence of structural alerts & \multicolumn{3}{|c|}{ Proceed with relevant assays (e.g. genotoxicity: AMES test, cardiotoxicity: hERG assay) } \\
\hline Toxicity in vivo & & \multicolumn{3}{|c|}{ Not overtly toxic in mice at the efficacious dose } \\
\hline \multirow[t]{3}{*}{ Chemical profile } & Chemical tractability & \multicolumn{3}{|c|}{ No potential liabilities which prevent scaled-up synthesis to $1 \mathrm{~g}+$} \\
\hline & $\begin{array}{l}\text { Structure-activity relationship } \\
\text { (SAR) }\end{array}$ & \multicolumn{3}{|c|}{ Preliminary SAR with minimum 10 compounds: avoid series lacking SAR or displaying 'flat' SAR } \\
\hline & Stereo-chemistry & \multicolumn{3}{|c|}{ Establish contribution to activity/toxicity/metabolism, ideally no chiral centre } \\
\hline
\end{tabular}


form of $L$. donovani, taking an activity cut-off of $10 \mu \mathrm{M}$ in dose-response determinations, calculated as $\mathrm{IC}_{50}$ combined with a 10 -fold selectivity index versus a mammalian cell line. This hit rate was obtained from screening approximately 500000 compounds from a variety of libraries, and is significantly lower than that from screening campaigns against the bloodstream form of $T$. brucei (average $0 \cdot 45 \%$, ranging from $0 \cdot 1-1 \cdot 2 \%$, approximately $1 \cdot 1$ million compounds assayed) as well as the intra-macrophagic form of $T$. cruzi (average $0 \cdot 30 \%$, ranging from $0 \cdot 05-0 \cdot 85 \%$, approximately 500000 compounds assayed). The relatively wide data distribution related to the assay hit rates can mostly be explained by the use of various assay protocols and screening laboratories to operate the screens as well as by the nature of the compound collections submitted to these assays as each library has been designed using a distinct set of criteria.

Such a low hit rate for intracellular $L$. donovani screening, compared to that of the extracellular blood stream form of T. brucei sp., is not surprising. For a compound to reach a target in an intracellular parasite it must first pass through two host cell membranes and often hostile environments for a drug-like compound, including a $\mathrm{pH}$ of 5.4 and a range of cell host lysosomal enzymes in the parasitophorous vacuole (Lang et al. 1994). Moreover, intracellular amastigotes of $L$. donovani are slow-replicating pathogens (De Rycker et al. 2013) compared to intracellular amastigotes of T. cruzi, and have developed very sophisticated strategies to survive using a reduced metabolism within the host cell defence system (Kima, 2007; Pescher et al. 2011). Also, by hiding in the vacuolar compartment it is difficult for drugs to reach the parasites and exert their effect on biochemical pathways or targets that are still functional and essential to the survival of the L. donovani amastigote pathogen.

Not all hit compounds which come out of the screening process continue through to lead optimization: a significant number are dropped following analysis and careful assessment of the identified molecules, due to chemical clustering, duplication with ongoing and past hit-to-lead and lead optimization programmes at DND $i$ or elsewhere, and incompatibility with the current internal target product profiles defined from those diseases (www.dndi.org/ diseases-projects/diseases.html). When the mechanism of action is not known, nor any preliminary structure-activity relationships associated with potency, it is usually safer to rely on hits associated with a high level of potency (ideally at the submicromolar level) in a phenotypic assay. Hit-to-lead and lead optimization experience has taught us that improving potency in the complex environments found in L. donovani or T. cruzi intracellular assays is a rather challenging task. Instead, optimization of $\mathrm{PK} /$ ADME is required to improve in vivo efficacy and build PK/PD knowledge within a specific series. Despite these hurdles, phenotypic screening is still considered more appropriate than target-based screening, and the fastest way to increase chemical diversity in a field where most targets are not fully validated and few known anti-infective classes of compounds are potent. To date, DND $i$ has identified an estimated total of over 50 chemical lead series from its screening and post-screening operations, with some, such as nitroimidazoles and oxaboroles, active against multiple protozoans. Two drug candidates emerging from this preclinical pipeline (www.dndi. org/diseases-projects/portfolio.html) are currently being progressed in clinical trials for human African trypanosomiasis (fexinidazole and SCYX-7158) and visceral leishmaniasis (fexinidazole).

\section{FUTURE PERSPECTIVES}

A large diversity within screened chemical libraries is most likely to yield the greatest number of new starting points for drug discovery. Currently, the major hurdle to this strategy is the technical (coupled to a financial) limitation in the capacity to screen against whole cell organisms, especially with respect to intracellular protozoa. Innovative approaches to improve throughput would relieve this bottleneck, and are currently under investigation. A recent manuscript (De Rycker et al. 2013) assessed the use of free living L. donovani axenic amastigotes as a surrogate predictive for intracellular amastigote activity. This comparative study demonstrated that from a screen of 16000 diverse compounds in both axenic and intra-macrophage assays, with reconfirmation of the axenic hits in an intracellular assay, the hits obtained from the intracellular screen were also identified in the axenic amastigote screen. The axenic pre-screen identified a large number of positives that did not translate to the intracellular screen (false positives) but reduced the size of the library for confirmatory screening by more than $95 \%$. Thus, a pre-screen against the extracellular form of the L. donovani parasite, followed by reconfirmation of the primary actives in the intracellular amastigote form of the assay, could offer a valuable cost-effective screening cascade when dealing with very large pharmaceutical company libraries. Such an approach could reasonably be applied to several global files from pharmaceutical companies. Indeed, despite difficulties in estimating the overlap in the chemical diversity between collections from Big Pharma, a recent precompetitive analysis of AstraZeneca ( 2.5 million) and Bayer Pharma AG ( 1.4 million) compound collections based on $2 \mathrm{D}$ near neighbour molecular fingerprinting highlighted only a limited overlap between the 2 compound sets (Kogej et al. 2013).

Screening larger diversity sets in association with an increasing capacity might not be the only way to 
secure new series for further development, however. At DND $i$ we intend to investigate, validate and eventually implement several alternative approaches to capture additional active scaffolds through an improved and more sophisticated selection of libraries. One attractive research avenue that we are currently exploring to significantly enhance the hit rate of screening programmes is the development of activitypredictive models, using public and proprietary data sets (the latter assumes partner's approval to use its IP in this framework has been secured). Such models have already been recently applied in the field of anti-infective discovery, notably in an Open Source context for tuberculosis and malaria (Periwal and Kishtapuram, 2012; Jamal and Periwal, 2013). Although is it still too early to come to any definite conclusions regarding this innovative approach, predictive modelling is expected to assist the prioritization of compounds to be submitted for screening and drastically reduce the related screening expenses, whilst increasing the hit rate and identification of novel active series.

The development of classification techniques to compare and visualize collections of compounds, such as through a list of descriptors related or unrelated to the chemical structure of the compound sets using Principal Component Analysis (Feher and Schmidt, 2003), without requesting the structural information to be shared among parties, could also be a valuable way to prioritize compound libraries for screening when collaborating with multiple pharmaceutical companies. Such tools could be used to share knowledge and identify potential opportunities to combine efforts at a later stage of drug development (e.g. across Product Development Partnerships preclinical programmes) without compromising confidentiality between the parties.

While obviously committed to honour any IP of its collaborating parties, DND $i$ also aims to share any derived knowledge with the scientific community active in the field of Neglected Diseases via its Open Innovation portal (www.dndi.org/diseases-projects/ open-innovation.html). Just as GSK has previously published the 13533 chemical libraries of compounds active against Plasmodium falciparum resulting from the screening of its 2 million compound library (Gamo et al. 2010), DND $i$ has also deposited various data sets in the public domain, from screening and lead programmes. These include the screening of the Medicines for Malaria Venture (MMV) malaria box (www.mmv.org/malariabox), an exciting and innovative initiative to boost drug discovery in the field of neglected disease. More data will follow as publication authorization is granted by the collaborating parties.

Finally, the few tractable lead series which have been identified to date should be fully exploited by using them to conduct in silico screens against diversity libraries from different sources. DND $i$ is working with a number of pharmaceutical companies to develop strategies to conduct such screens against their libraries with each using their own proprietary screening algorithms and avoiding contamination of their intellectual property. This should maximize the diversity of scaffolds and annotation around a single series identified from a phenotypic screen.

\section{ACKNOWLEDGEMENTS}

The authors would like to thank Dr Sue Wells for the critical reading of the manuscript.

\section{FINANCIAL SUPPORT}

The Drugs for Neglected Diseases initiative (DND $i$ ) is grateful to the following donors for funding its screening programmes from 2006 to date: Spanish Agency for International Development Cooperation (AECID), Spain; Department for International Development (DFID), United Kingdom; Ministry of Foreign and European Affairs (MAEE), France; Federal Ministry of Education and Research (BMBF) through KfW, Germany; GTZ on behalf of the Government of the Federal Republic of Germany; Swiss Agency for Development and Cooperation (SDC), Switzerland; Dutch Ministry of Foreign Affairs (DGIS), The Netherlands; Sasakawa peace Foundation; UBS Optimus Foundation; Sandoz Family Foundation; Medecins Sans Frontières (Doctors without Borders); Bill \& Melinda Gates Foundation, United States of America. The donors had no role in study design, data collection and analysis, decision to publish, or preparation of the manuscript. The authors have no other relevant affiliations or financial involvement with any organization or entity with a financial interest in or financial conflict with the subject matter or materials discussed in the manuscript apart from those disclosed. No writing assistance was utilized in the production of this manuscript.

\section{REFERENCES}

Bell, A. S., Bradley, J., Everett, J. R., Knight, M., Loesel, J., Mathias, J., McLoughlin, D., Mills, J., Sharp, R. E., Williams, C. and Wood, T. P. (2013). Plate-based diversity subset screening: an efficient paradigm for high throughput screening of a large screening file. Molecular Diversity 17, 319-335. doi: 10.1007/s11030-013-9438-x.

Castillo, E. A., Dea-Ayuela, M., Bolas-Fernandez, F., Rangel, M. E. and Gonzalez-Rosende, M. (2010). The kinetoplastid chemotherapy revisited: current drugs, recent advances and future perspectives. Current Medicinal Chemistry 17, 4027-4051.

Chawla, B. and Madhubala, R. (2010). Drug target in Leishmania. Fournal of Parasitology Disease 34, 1-13.

Das, A., Dasgupta, A., Sengupta, T. and Majumder, H. K. (2004). Topoisomerases of kinetoplastid parasites as potential chemotherapeutic targets. Trends in Parasitology, 20, 381-387.

De Rycker, M., Hallyburton, I., Thomas, J., Campbell, L., Wyllie, S., Joshi, D., Cameron, S., Gilbert, I. H., Wyatt, P. G., Frearson, J. A., Fairlamb, A.H. and Gray, D.W. (2013). Comparison of a highthroughput high-content intracellular Leishmania donovani assay with an axenic amastigote assay. Antimicrobial Agents and Chemotherapy 57, 2913-2922. doi: 10.1128/AAC.02398-12.

Feher, M. and Schmidt, J. M. (2003). Property distributions: differences between drugs, natural products, and molecules from combinatorial chemistry. Fournal of Chemical Information and Modeling 43, 218-227.

Frearson, J. A., Brand, S., McElroy, S. P., Cleghorn, L. A., Smid, O., Stojanovski, L., Price, H.P., Guther, M. L., Torrie, L. S., Robinson, D. A., Hallyburton, I., Mpamhanga, C. P., Brannigan, J. A., Wilkinson, A. J., Hodgkinson, M., Hui, R., Qiu, W., Raimi, O. G., van Aalten, D. M., Brenk, R., Gilbert, I. H., Read, K. D., 
Fairlamb, A. H., Ferguson, M. A., Smith, D. F. and Wyatt, P. G. (2010). $N$-myristoyltransferase inhibitors as new leads to treat sleeping sickness. Nature 464, 728-732. doi: 10.1038/nature08893.

Frearson, J. A., Wyatt, P. G., Gilbert, I. H. and Fairlamb, A. H. (2007). Target assessment for antiparasitic drug discovery. Trends in Parasitology 23, 589-595.

Gamo, F. J., Sanz, L. M., Vidal, J., de Cozar, C., Alvarez, E., Lavandera, J. L., Vanderwall, D. E., Green, D. V., Kumar, V., Hasan, S., Brown, J. R., Peishoff, C. E., Cardon, L. R. and Garcia-Bustos, J. F. (2010). Thousands of chemical starting points for antimalarial lead identification. Nature 465, 305-310. doi: 10.1038/ nature09107.

Ioset, J.-R. and Chang, S. (2011). Drugs for neglected diseases initiative model of drug development for neglected diseases: current status and future challenges. Future Medicinal Chemistry 1, 1361-1371. doi: 10.4155/ fmc.11.102.

Jamal, S. and Periwal, V. (2013). Open source drug discovery consortium, Scaria V. Predictive modeling of anti-malarial molecules inhibiting apicoplast formation. BMC Bioinformatics 15, 55-62. 14:55. doi: 10.1186/ 1471-2105-14-55.

Keller, T.H., Shi, P. Y. and Wang, Q.Y. (2011). Anti-infectives: can cellular screening deliver? Current Opinion in Chemical Biology 15, 529-533. doi: 10.1016/j.cbpa.2011.06.007.

Kima, P.E. (2007). The amastigote forms of Leishmania are experts at exploiting host cell processes to establish infection and persist. International Fournal for Parasitology 37, 1087-1096.

Kogej, T., Blomberg, N., Greasley, P. J., Mundt, S., Vainio, M. J., Schamberger, J., Schmidt, G. and Hüser, J. (2013). Big pharma screening collections: more of the same or unique libraries? The AstraZeneca-Bayer Pharma AG case. Drug Discovery Today http://dx.doi. org/10.1016/j.drudis.2012.10.011.

Lang, T., Hellio, R., Kaye, P. M. and Antoine, J. C. (1994). Leishmania donovani-infected macrophages: characterization of the parasitophorous vacuole and potential role of this organelle in antigen presentation. Fournal of Cell Science 107, 2137-2150.
McKerrow, J. H., Rosenthal, P. J., Swenerton, R. and Doyle, P. (2008). Development of protease inhibitors for protozoan infections. Current Opinion in Infectious Diseases 21, 668-672. doi: 10.1097/ QCO.0b013e328315cca9.

Payne, D. J., Gwynn, M. N., Holmes, D. J., and Pompliano, D. L. (2007). Drugs for bad bugs: confronting the challenges of antibacterial discovery. Nature Reviews Drug Discovery 6, 29-40.

Periwal, V. and Kishtapuram, S. (2012). Open Source Drug Discovery Consortium, Scaria V. Computational models for in-vitro antitubercular activity of molecules based on high-throughput chemical biology screening datasets. BMC Pharmacology 31, 1-7. 12:1. doi: 10.1186/14712210-12-1.

Pescher, P., Blisnick, T., Bastin, P. and Spath, G. F. (2011). Quantitative proteome profiling informs on phenotypic traits that adapt Leishmania donovani for axenic and intracellular proliferation. Cell Microbiology 13 978-991. doi: 10.1111/j.1462-5822.2011.01593.x.

Renslo, A. R. and McKerrow, J.H. (2006). Drug discovery and development for neglected parasitic diseases. Nature Chemical Biology 2, 701-710.

Sams-Dodd, F. (2005). Target-based drug discovery: is something wrong? Drug Discovery Today 10,139-147.

Siqueira-Neto, J. L., Moon, S., Jang, J., Yang, G., Lee, C., Moon, H. K., Chatelain, E., Genovesio, A., Cechetto, J. and Freitas-Junior, L. H (2012). An image-based high-content screening assay for compounds targeting intracellular Leishmania donovani amastigotes in human macrophages. PLoS Neglected Tropical Diseases 6, e1671. doi: 10.1371/journal. pntd.0001671.

Sykes, M. L. and Avery, V. M. (2009). Development of an Alamar Blue viability assay in 384-well format for high throughput whole cell screening of Trypanosoma brucei brucei bloodstream form strain 427. American Fournal of Tropical Medicine and Hygiene 81, 665-674. doi: 10.4269/ ajtmh.2009.09-0015.

Wyatt, P. G., Gilbert, I. H., Read, K. D. and Fairlamb, A. H. (2011) Target validation: linking target and chemical properties to desired product profile. Current Topics in Medicinal Chemistry 11, 1275-1283. 